\title{
Statistics at the tip of a branching random walk and the delay of traveling waves
}

\author{
É. Brunet ${ }^{1}$ and B. Derrida ${ }^{1}$ \\ ${ }^{1}$ Laboratoire de Physique Statistique, École Normale Supérieure, UPMC Paris 6, \\ Université Paris Diderot, CNRS, 24 rue Lhomond, 75005 Paris, France
}

\begin{abstract}
We study the limiting distribution of particles at the frontier of a branching random walk. The positions of these particles can be viewed as the lowest energies of a directed polymer in a random medium in the mean-field case. We show that the average distances between these leading particles can be computed as the delay of a traveling wave evolving according to the Fisher-KPP front equation. These average distances exhibit universal behaviors, different from those of the probability cascades studied recently in the context of mean field spin-glasses.
\end{abstract}

PACS numbers: 02.50.-r, 05.40.-a, 89.75.Hc

The interest for branching random walks has a long history in Mathematics 1, 2, 3, Physics and Biology. In Biology they are commonly used to model the genealogies of evolving populations, the spread of an advantageous gene or of an infection, the combined effects of selection and mutations [4, 5, 6]. In Physics they also appear in many contexts such as reaction-diffusion models 7 , 8, particle physics 9, 10, or the theory of disordered systems [11, 12].

In one dimension, the right frontier of a branching random walk is the region located near its rightmost particle. An interesting question is what does the branching random walk look like when seen from this frontier. For example one can try to determine the position of the second, the third, $\ldots$ or the $n^{\text {th }}$ rightmost particle in the frame of the first rightmost particle. The statistical properties of these positions depend on time and have a well defined long time limit 3 . which we study in this letter using traveling wave equations of the Fisher-KPP type [4, 13, 14

$$
\frac{\partial h}{\partial t}=\frac{\partial^{2} h}{\partial x^{2}}+h-h^{2}
$$

The fluctuating distances between these rightmost particles allows one to understand why directed polymers in a random medium [1] have non-selfaveraging properties similar to mean field spin-glasses [15]. Their study is also motivated by the growing interest for the statistics of extreme events [12, 16, 17, 18, 19, 20, 21, 22] which dominate a number of physical processes 223, 24]. The last two decades have seen the emergence of universal statistical properties of the probability cascades describing the energies of the low lying states of several spin-glass models [22, 25, 26, 27, 28, 29, 30. Somewhat surprinsingly, as shown below, the distribution of the distances between the extreme positions of particles in a branching random walk (which are nothing but the energies of the low lying states in the mean field version of directed polymer problem [11]) is different from the predictions of the probability cascades [22, 25, 26, 27, 28, 29, 30.

To start with a simple case, we consider a continuous time branching random walk in one dimension: at time $t=0$ there is a single particle at the origin $x=0$. This particle diffuses (for convenience we normalize the variance of its displacement during time $t$ to be $2 t$ ) and branches at rate 1 . Whenever a branching event occurs, the offspring become themselves branching random walks which diffuse and branch with the same rates. The number of particles grows exponentially with time and they occupy a region which grows linearly with time 1, 2.

It has been known for a long time [1, 2, 12, that the distribution of the rightmost particle of a branching random walk can be determined by solving a traveling wave equation: the probability $Q_{0}(x, t)$ that, at time $t$, there is no particle at the right of of $x$, satisfies

$$
\frac{\partial Q_{0}}{\partial t}=\frac{\partial^{2} Q_{0}}{\partial x^{2}}+Q_{0}^{2}-Q_{0}
$$

[The derivation of (2) is standard: one decomposes the time interval $(0, t+d t)$ into two intervals $(0, d t)$ and $(d t, t+d t)$, and write that $Q_{0}(x, t+d t)=Q_{0}(x, t)^{2} d t+$ $\left\langle Q_{0}(x-\eta, t)\right\rangle_{\eta}(1-d t)$ where the first term represents the contribution of a branching event and $\eta$ in the second term the displacement due to diffusion during the first time interval $(0, d t)$. With our normalization $\left\langle\eta^{2}\right\rangle=2 d t$.]

Up to the change $h=1-Q_{0}$, equation (2) is the FisherKPP equation (1). Since at time $t=0$ there is a single particle at the origin, the initial condition is simply

$$
Q_{0}(x, 0)=1 \text { for } x>0, \quad Q_{0}(x, 0)=0 \text { for } x<0 .
$$

If $Q_{n}(x, t)$ is the probability that there are exactly $n$ particles on the right of $x$, one can see, as for $Q_{0}$, that the generating function $\psi_{\lambda}(x, t)$, defined as

$$
\psi_{\lambda}(x, t)=\sum_{n \geq 0} \lambda^{n} Q_{n}(x, t),
$$

evolves according to the same equation (2), the only difference being that the initial condition is replaced by

$$
\psi_{\lambda}(x, 0)=1 \text { for } x>0, \quad \psi_{\lambda}(x, 0)=\lambda \text { for } x<0 .
$$

We are now going to see that the knowledge of $\psi_{\lambda}(x, t)$ allows one to obtain the average distances between the 
rightmost particles of the system. If $p_{n}(x, t)$ is the probability of finding the $n^{\text {th }}$ rightmost particle at position $x$, it is easy to see that

$$
\frac{\partial Q_{0}}{\partial x}=p_{1}(x, t) \text { and } \frac{\partial Q_{n}}{\partial x}=p_{n+1}(x, t)-p_{n}(x, t) .
$$

The average position $\left\langle X_{n}(t)\right\rangle$ of the $n^{\text {th }}$ rightmost particle and the average distance $\left\langle d_{n, n+1}(t)\right\rangle$ between the $n^{\text {th }}$ and $(n+1)^{\text {th }}$ rightmost particles can then be defined by

$$
\begin{array}{r}
\left\langle X_{n}(t)\right\rangle=\int x p_{n}(x, t) d x, \\
\left\langle d_{n, n+1}(t)\right\rangle=\left\langle X_{n}(t)\right\rangle-\left\langle X_{n+1}(t)\right\rangle .
\end{array}
$$

[One should notice that the normalization of $p_{n}(x, t)$ is not 1 but $\int p_{n}(x, t) d x=\left(1-e^{-t}\right)^{n-1}$ due to the events for which the total number of particles at time $t$ is still less than $n$. One could prefer to use different definitions of the positions or of the distances, for example by conditioning on the fact that there are at least $n+1$ particles in the system, but any such definition would coincide with $(7 / 8)$ up to contributions which decay exponentially with time and disappear in the long time limit that we study below.]

With the definition (8) we obtain from $(4677)$ that

$$
\sum_{n \geq 1} \lambda^{n}\left\langle d_{n, n+1}(t)\right\rangle=\int x\left[\frac{d Q_{0}}{d x}-\frac{d \psi_{\lambda}}{d x}\right] d x,
$$

which relates the distances $\left\langle d_{n, n+1}(t)\right\rangle$ between the rightmost particles to the solution $\psi_{\lambda}(t)$ of the partial differential equation (2) with the initial condition (5).

We have integrated numerically the equations satisfied by $\psi_{\lambda}(x, t)$ and its derivatives with respect to $\lambda$ to measure the distances $\left\langle d_{n, n+1}(t)\right\rangle$ between the $n^{\text {th }}$ and $(n+1)^{\text {th }}$ rightmost particles. In our numerical integration, we had to discretize space and time; we checked that our results shown in figure 1 were stable when we decreased our integration steps.
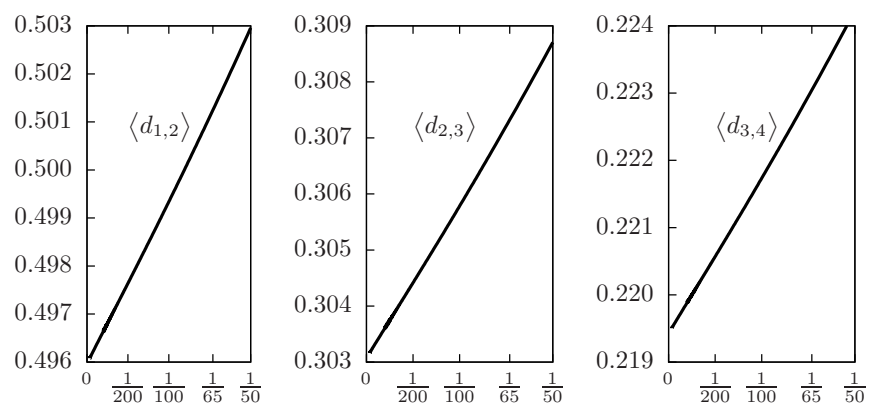

FIG. 1: The average distances between the first rightmost particles $\left\langle d_{1,2}(t)\right\rangle,\left\langle d_{2,3}(t)\right\rangle$ and $\left\langle d_{3,4}(t)\right\rangle$ of a branching random walk versus $1 / t$, for $t$ up to 3000 .

One can first remark that, in contrast to standard Monte-Carlo simulations, where all the branching events would be simulated and for which the maximum reachable time would be $t \sim 20$ (with a number of particles $e^{t}$ of order $10^{9}$ ), the integration of 2 or of its derivatives allows one to achieve much larger times. One can also notice in figure 1 that the distances converge like $1 / t$ to well defined values. We will see that this $1 / t$ convergence is consistent with our analytic expression (24) below.

We did not find an analytic theory to predict the limiting values that we measured as in figure 1 .

$$
\begin{aligned}
& \left\langle d_{1,2}\right\rangle \simeq 0.496, \quad\left\langle d_{2,3}\right\rangle \simeq 0.303, \quad\left\langle d_{3,4}\right\rangle \simeq 0.219 \\
& \left\langle d_{4,5}\right\rangle \simeq 0.172, \quad\left\langle d_{5,6}\right\rangle \simeq 0.142, \quad\left\langle d_{6,7}\right\rangle \simeq 0.121 .
\end{aligned}
$$

As shown below (17), we can however predict their large $n$ behavior.

Before doing so, it is interesting to compare our results (10) to the expected values of the gaps between the low lying energies of spin-glass models such as the REM and the GREM [31, 32. In these models one can show that these energies are given by probability cascades 22,25 , 27, 28, 29] and that the energy gaps at the leading edge are the same as those of a Poisson process on the line with an exponential density. For such a process, with density $e^{-\alpha x}$, the probability distribution of the positions is $p_{n}(x)=\exp \left[-n \alpha x-e^{-\alpha x} / \alpha\right]$ from which one gets through $(78)$

$$
\left\langle d_{n, n+1}\right\rangle_{\mathrm{GREM}}=\frac{1}{\alpha n} .
$$

Clearly there is no choice of $\alpha$ for which our numerical results (10) are compatible with (11).

It is well known [2, 14 that the solution $Q_{0}(x, t)$ of the Fisher-KPP equation (2) with the step initial condition (3) becomes, for large $t$, a traveling wave of the form

$$
Q_{0}(x, t) \simeq F\left[x-\left\langle X_{1}(t)\right\rangle\right],
$$

where the shape $F(z)$ of the front $[F(z) \rightarrow 1$ as $z \rightarrow \infty$ and $F(z) \rightarrow 0$ as $z \rightarrow-\infty$ ] is time-independent and its position, which can be defined as the average position $\left\langle X_{1}(t)\right\rangle$ of the rightmost particle, has the following long time behavior [2, 14, 33.

$$
\left\langle X_{1}(t)\right\rangle=2 t-\frac{3}{2} \ln t+\mathcal{O}(1) .
$$

$\psi_{\lambda}(x, t)$ is also the solution of the Fisher-KPP equation (2), but with the initial condition (5). As $\psi_{\lambda}(x, 0)$ decays fast enough [14, one expects the same large $t$ behavior as $12[13)$, up to a $\lambda$-dependent delay $f(\lambda)$ due to the change of initial condition:

$$
\psi_{\lambda}(x, t) \simeq F\left[x-\left\langle X_{1}(t)\right\rangle+f(\lambda)\right] .
$$

From $\left.\begin{array}{l|l|l|l|l|l|l}9 & 12 & 14\end{array}\right)$ we see that the translation $f(\lambda)$ is nothing but the generating function of the average distances

$$
f(\lambda)=\lim _{t \rightarrow \infty} \sum_{n \geq 1} \lambda^{n}\left\langle d_{n, n+1}(t)\right\rangle .
$$


We were not able to find an analytic expression of the delay function $f(\lambda)$ for arbitrary $\lambda$. For $\lambda$ close to 1 , however, we are going to show that

$$
f(\lambda)=\tau_{\lambda}-\ln \tau_{\lambda}+\mathcal{O}(1) \quad \text { with } \tau_{\lambda}=-\ln (1-\lambda) .
$$

This implies 15 that the distances have the following large $n$ asymptotics

$$
\left\langle d_{n, n+1}(t)\right\rangle_{t=\infty} \simeq \frac{1}{n}-\frac{1}{n \ln n}+\cdots
$$

Compared with (11), we see that there is a correction, which we believe to be universal as discussed below. (Note that the same asymptotic distances would be obtained for uncorrelated particles distributed according to a Poisson point process with a density $-x e^{-x}$ for negative $x$.)

For $\lambda$ close to 1 , the time $\tau_{\lambda}$ in 16 is the characteristic time it takes $\psi_{\lambda}(-\infty, t)$ to reach a value close to 0 . The most naïve idea to derive (16) would be to say that it takes this time $\tau_{\lambda}$ for $\psi_{\lambda}(x, t)$ to look like the step function $Q_{0}(x, 0)$, and then to start moving like $Q_{0}(x, t)$. As the asymptotic velocity is 2 (see (13)) this would lead to a delay $f(\lambda) \simeq 2 \tau_{\lambda}$ which is wrong (see 160) by a factor 2 . The problem with this idea is that, while $\psi_{\lambda}(x, t)$ evolves to approach 0 on the negative $x$ axis, a tail builds up on the positive $x$ axis which has a strong influence on the dynamics later on.

To derive (16), we need to understand the shape of $\psi_{\lambda}(x, t)$ for $t>\tau_{\lambda}$. Let $Y_{t}$ be the position where $\psi_{\lambda}\left(Y_{t}, t\right)=1 / 2$. We have checked both numerically and analytically that the following picture holds for $t$ and $\tau_{\lambda}$ large, with a given ratio $t / \tau_{\lambda}$ larger than 1 :

In the range where $x-Y_{t}$ is of order 1

$$
\psi_{\lambda}(x, t) \simeq \phi_{v(t)}\left(x-Y_{t}\right),
$$

where $v(t)=\dot{Y}_{t}$ is the instaneous velocity of the front and $\phi_{v}$ is the solution of the Fisher-KPP equation moving at a constant velocity $v$, i.e. the solution of

$$
\phi_{v}^{\prime \prime}+v \phi_{v}^{\prime}+\phi_{v}^{2}-\phi_{v}=0
$$

with $\phi_{v}(-\infty)=0$ and $\phi_{v}(+\infty)=1$. (The same form (18) is used in 34.) For definiteness, we normalize such that $\phi_{v}(0)=1 / 2$. This determines a unique solution which has, if $v>2$, the following asymptotics for $z \rightarrow+\infty$ :

$$
1-\phi_{v}(z) \simeq B_{\gamma} e^{-\gamma z}+o\left(e^{-\gamma z}\right),
$$

where $\gamma$ is the smallest solution of

$$
v=\gamma+\gamma^{-1} .
$$

On the other hand, in the range $x-Y_{t} \gg 1, \psi_{\lambda}(x, t)$ is accurately given by the solution of the equation obtained by linearizing (2) around 1 :

$$
1-\psi_{\lambda}(x, t) \simeq \frac{(1-\lambda) e^{t}}{\sqrt{\pi}} \int_{x / \sqrt{4 t}}^{\infty} e^{-u^{2}} d u .
$$

Then, using the asymptotics of the error function $\int_{X}^{\infty} \exp \left(-u^{2}\right) d u \simeq \exp \left(-X^{2}\right) /(2 X)$ and requiring that \begin{tabular}{l|l|}
18 & 20
\end{tabular} and 22 match in the range $1 \ll x-Y_{t} \ll \sqrt{t}$, one gets that $\gamma(t)$ and $Y_{t}$ should satisfy

$$
B_{\gamma(t)} e^{-\gamma(t)\left(x-Y_{t}\right)} \simeq \frac{(1-\lambda) e^{t} \sqrt{t}}{Y_{t} \sqrt{\pi}} e^{-\frac{Y_{t}^{2}}{4 t}-\frac{Y_{t}\left(x-Y_{t}\right)}{2 t}} .
$$

To match the dependence in $x-Y_{t}$, we need $Y_{t} \simeq 2 t \gamma(t)$ to first order. Then matching the prefactors leads to

$$
\begin{gathered}
Y_{t} \simeq 2 \sqrt{t\left(t-\tau_{\lambda}\right)}-\frac{\ln t}{2 \gamma(t)}-\frac{\ln \left[2 \sqrt{\pi} \gamma(t) B_{\gamma(t)}\right]}{\gamma(t)}, \\
\text { with } \quad \gamma(t) \simeq \sqrt{\frac{t-\tau_{\lambda}}{t}} .
\end{gathered}
$$

Note that the relation (21) is indeed satisfied to leading order as $\dot{Y}_{t} \simeq \gamma(t)+\gamma(t)^{-1}$. In figure 2 we see that the agreement between the leading term in (24) and the position obtained by integrating numerically (2) with the initial condition (5) is quite good. One could also see in (24) a $1 / t$ convergence of the $\lambda$-dependent delay which is consistent with the numerical results of figure 1 .

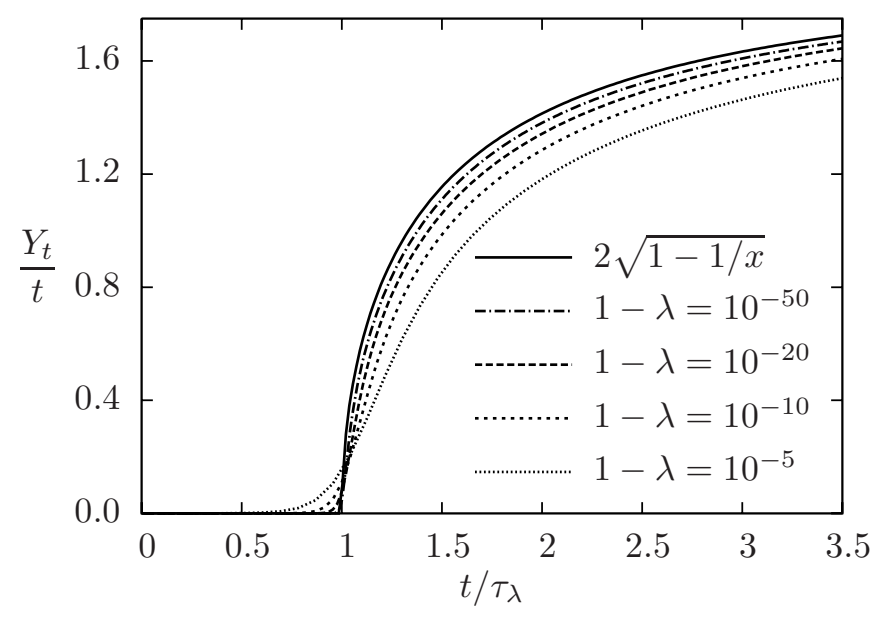

FIG. 2: The prediction 24 to the leading order for the position of the front is compared to the position measured by integrating (2) with the initial condition (5). As in (16), $\tau_{\lambda}=-\ln (1-\lambda)$.

For $t / \tau_{\lambda}$ large, $\gamma(t) \rightarrow 1$ and $v(t) \rightarrow 2$. For $v=2$, the solution of (19) satisfies $1-\phi_{2}(z) \simeq A z e^{-z}$ for large $z$ [14, 33. For $v$ slightly larger than 2 , the next term in the large $z$ expansion 20 is $1-\phi_{v}(z) \simeq B_{\gamma} e^{-\gamma z}+$ $C_{\gamma} e^{-z / \gamma}+o\left(e^{-z / \gamma}\right)$. For consistency in the limit $v \rightarrow 2$, one has

$$
B_{\gamma} \simeq-C_{\gamma} \simeq \frac{A}{2(1-\gamma)} \quad \text { as } \gamma \rightarrow 1,
$$

so that $B_{\gamma(t)} \simeq A t / \tau_{\lambda}$ from 25260. Thus, (24) becomes $Y_{t} \simeq 2 t-\tau_{\lambda}-(3 / 2) \ln t+\ln \tau_{\lambda}+\mathcal{O}(1)$, which gives $13 \mid 14$ 16. 
One can repeat everything if, instead of starting with a single particle at the origin, one starts with $K$ of particles at positions $y_{1}, \ldots, y_{K}$. One simply needs to replace $\psi_{\lambda}(x, t)$ defined in (4) by $\prod_{1<i<K} \psi_{\lambda}\left(x-y_{i}, t\right)$, with a similar change for $Q_{0}(x, t) \equiv \bar{\psi}_{0}(x, t)$. As a result, in the long time limit, the delay function $f(\lambda)$, and therefore the distances between the rightmost particles remain unchanged. This property is remarkable: whatever the positions of the initial particles are (as long as there are a finite number of them) the limiting average distances and probably the whole limiting measure seen from the rightmost particle are the same.

We can also extend all our calculations to more general branching random walks. For example one may consider a discrete time case where at each time step, every particle splits into $K$ new particles, and the position of each new particle is shifted from its parent by a random amount $\epsilon$ drawn from a given distribution $\rho(\epsilon)$. Apart from a few changes, such as 21 which is replaced by

$$
v=\gamma^{-1} \ln \left[K \int \rho(\epsilon) e^{\gamma \epsilon} d \epsilon\right]
$$

$\tau_{\lambda}$ in 16 which becomes $-\ln (1-\lambda) / \ln K$ or $\gamma(t)$ in 25 which becomes the solution of

$$
\gamma^{2} \frac{d v}{d \gamma}=-\frac{\ln (1-\lambda)}{t}
$$

everything remains unchanged. In particular 16 17) are simply divided by the value $\gamma_{0}$ of $\gamma$ which minimizes the expression (27) of $v$. Thus, the asymptotics of both the delay (16) and the distances (17) look universal, up to a scale factor $\gamma_{0}$.

In the present letter we have seen that the distances between the rightmost particles at the frontier of a branching random walk have statistical properties 10 17) which can be understood as the delay 14 15 16 of a traveling wave. Other properties, such as the correlations of these distances or even their whole probability distribution can also be understood in terms of the delay of a traveling wave. For example if $R_{n, m}(x, y, t)$ is the probability that there are $n$ particles at the right of $x$ and $m$ particles at the right of $y$, on can show that

$$
\left\langle d_{n, n+1}(t) d_{m, m+1}(t)\right\rangle=\int d x x \int d y y \frac{\partial^{2} R_{n, m}(x, y, t)}{\partial x \partial y}
$$

while the generating function $\sum_{n, m} \lambda^{n} \mu^{m} R_{n, m}(x, x+c, t)$ defined as in (4) evolves according to the Fisher-KPP equation (2) with a new initial condition.

A surprising aspect of the present work is that the statistics of the leading particles, in the long time limit, do not depend on the positions or on the number of particles we start with, as long as there is a finite number of them. This means that the limiting measure has the following stability property: if one takes two realizations of the leading particles according to this measure and shifts one of them by an arbitrary amount, then the superimposition of these two realizations gives a new realization of the same measure, up to a translation.

[1] H. P. McKean, Comm. Pure Appl. Math. 28, 323 (1975).

[2] M. D. Bramson, Comm. Pure Appl. Math. 31, 531 (1978).

[3] S. P. Lalley and T. Sellke, Ann. Prob. 15, 1052 (1987).

[4] R. A. Fisher, Annals of Eugenics 7, 355 (1937).

[5] D. A. Kessler, H. Levine, D. Ridgway, and L. Tsimring, J. Stat. Phys. 87, 519 (1997).

[6] I. Golding, Y. Kozlovsky, I. Cohen, and E. Ben-Jacob, Physica A 260, 510 (1998).

[7] C. R. Doering, C. Mueller, and P. Smereka, Physica A 325, 243 (2003).

[8] A. De Masi, P. Ferrari, and J. Lebowitz, J. Stat. Phys. 44, 589 (1986).

[9] S. Munier, Phys. Rep. 473, 1 (2009).

[10] R. Peschanski, Nucl. Phys. B 805, 377 (2008).

[11] B. Derrida and H. Spohn, J. Stat. Phys. 51, 817 (1988).

[12] D. S. Dean and S. N. Majumdar, Phys. Rev. E 64, 046121 (2001).

[13] A. Kolmogorov, I. Petrovsky, and N. Piscounov, Bull. Univ. État Moscou, A 1, 1 (1937).

[14] W. van Saarloos, Phys. Rep. 386, 29 (2003).

[15] M. Mézard, G. Parisi, N. Sourlas, G. Toulouse, and M. A. Virasoro, Journal de Physique 45, 843 (1984).

[16] C. A. Tracy and H. Widom, Comm. Math. Phys. 159, 151 (1994).

[17] J.-P. Bouchaud and M. Mézard, J. Phys. A 30, 7997 (1997).

[18] T. W. Burkhardt, G. Györgyi, M. R. Moloney, and Z. Racz, Phys. Rev. E 76, 041119 (2007).

[19] G. Györgyi, M. R. Moloney, K. Ozogany, and Z. Racz, Phys. Rev. Lett. 100, 210601 (2008).

[20] S. N. Majumdar and P. Krapivsky, Phys. Rev. E 65, 036127 (2002).

[21] S. Sabhapandit and S. N. Majumdar, Phys. Rev. Lett. 98, 140201 (2007).

[22] A. Ruzmaikina and M. Aizenman, Ann. Prob. 33, 82 (2005).

[23] F. Igloi and C. Monthus, Phys. Rep. 412, 277 (2005).

[24] L. P. Kadanoff, J. Stat. Phys. 122, 1293 (2006).

[25] D. Ruelle, Comm. Math. Phys 108, 225 (1987).

[26] E. Bolthausen and A.-S. Sznitman, Comm. Math. Phys. 197, 247 (1998).

[27] M. Aizenman, R. Sims, and S. L. Starr, Contemporary Math. Series 437, 1 (2007).

[28] L. P. Arguin, J. Stat. Phys. 126, 951 (2007).

[29] A. Bovier and I. Kurkova, Comm. Math. Phys. 263, 535 (2006).

[30] A. Bovier and I. Kurkova, J. Stat. Phys. 126, 933 (2007).

[31] B. Derrida, Phys. Rev. B 24, 2613 (1981).

[32] B. Derrida, J. Phys. Lett. 46, L401 (1985).

[33] É. Brunet and B. Derrida, Phys. Rev. E 56, 2597 (1997).

[34] U. Ebert and W. van Saarloos, Phys. Rev. Lett. 80, 1650 (1998). 